\title{
Effect of Reimbursement Reductions on Bone Mineral Density Testing for Female Medicare Beneficiaries
}

\author{
Ji Won Yoo, M.D., M.Sc., ${ }^{1,2}$ Shunichi Nakagawa, M.D., ${ }^{3,4}$ and Sulgi Kim, Ph.D. ${ }^{5}$
}

\begin{abstract}
Background: We examined whether the recent reimbursement reductions on the bone mineral density (BMD) test affected BMD testing in female Medicare beneficiaries with or without supplemental private health insurance. Methods: Retrospectively analyzing hospital administrative and clinical data on female Medicare beneficiaries $(n=1320)$, we reviewed whether participants received BMD testing before (January 2004-December 2006) or after (January 2007-December 2009) reimbursement reductions for BMD testing. After adjusting for demographics and clinical characteristics, we performed Cox proportional hazard regression analyses of the BMD test including data from all study participants; we then performed separate regression analyses using data with or without supplemental private health insurance.

Results: In those without supplemental private health insurance $(n=421)$, less frequent BMD testing occurred after reimbursement reductions for BMD testing (hazard ratio [HR] 0.67, 95\% confidence intervals [CI] 0.34-0.98; $p=0.03)$. By contrast, in the overall participants $(n=1320)$ and those with supplemental private health insurance $(n=899)$, the number of BMD tests did not change significantly after reimbursement reductions for BMD testing. Conclusions: We found a significant association between reimbursement reductions and decrease in BMD tests in female Medicare beneficiaries without supplemental private health insurance.
\end{abstract}

\section{Introduction}

$\mathrm{O}$ STEOPOROSIS IS CHARACTERIZED BY LOW BONE MASS and is a progressive, systemic disease that causes bone fragility and fracture. ${ }^{1-3}$ About one of every two white women will experience an osteoporosis-related fracture at some point in her lifetime. ${ }^{2}$ The number of persons at risk is increasing as the population ages. Detection of osteoporosis during treatable stages of the disease is optimal; early detection and treatment have been shown to decrease associated fracture, nursing home placement, and mortality. ${ }^{2-4}$ Dual-energy $\mathrm{x}$-ray absorptiometry (DXA) is currently the gold standard for measuring bone mineral density (BMD). ${ }^{2,4-6}$ Medicare Part B reimburses DXA coverage once every 24 months for women who have one or more clinical risk factors, such as old age. ${ }^{5,6}$ In agreement with U.S. Preventive Service Task Force (USPSTF) recommendations, the National Osteoporosis Foundation (NOF) advises DXA testing of all women aged $\geq 65$ at least once every 24 months. ${ }^{5-7}$
Since Medicare began to reduce reimbursement for DXA tests performed in nonhospital settings (e.g., independent diagnostic facilities and physicians' offices) from an average of $\$ 139$ in 2006 to $\$ 72$ in 2009 , the parallel attenuation of earlier growth in total number of DXA tests performed at these facilities has occurred..$^{8-10}$ Elderly women with higher incomes or supplemental drug coverage insurance were reported to be better able to access DXA testing. ${ }^{11}$ Another study reflecting the attitudes of private health insurance holders reported that the use of DXA tests grew at a slower rate since reimbursement cuts for such tests. ${ }^{12}$ However, no studies examining how the effects of reimbursement reductions on the use of DXA vary by supplemental health insurance status have yet been reported. Elderly women are the most common demographic group to have osteoporosis and therefore, are expected to be more vulnerable to reimbursement reductions than any other age group. ${ }^{2-4}$ The present study examines whether the effects of reimbursement reductions on BMD testing vary by supplemental private health insurance for female Medicare beneficiaries.

\footnotetext{
${ }^{1}$ Department of Internal Medicine, University of Michigan Medical School, Ann Arbor, Michigan.

${ }^{2}$ Geriatric Research, Education and Clinical Center, Veterans Affairs Ann Arbor Healthcare System, Ann Arbor, Michigan.

${ }^{3}$ Department of Geriatrics and Palliative Medicine, Mount Sinai School of Medicine, New York, New York.

${ }^{4}$ Geriatric Research, Education and Clinical Center, James J. Peters Veterans Affairs Medical Center, Bronx, New York.

${ }^{5}$ Department of Epidemiology, School of Public Health, University of Washington, Seattle, Washington.
} 


\section{Materials and Methods}

\section{Study design and study participants}

The study site is a U.S. urban nonprofit academic healthcare system. The total study period was 6 years, 3 years before (phase 1, January 2004-December 2006) and 3 years after (phase 2, January 2007-December 2009) reimbursement reductions. Because Medicare reimburses DXA test at intervals of $\geq 2$ years and the median intervals between serial DXA tests were reported to be 2.2 years in the prior literature, we determined 3 years as the period of each study phase. ${ }^{5,13} \mathrm{In}$ clusion criteria were (1) female Medicare Parts A and B beneficiaries (hence, aged $\geq 65$ ) for at least 12 months before study enrollment, (2) at least one clinic visit before study enrollment (surrogate marker for continuity of care), and (3) women without a history of osteoporosis at the time of study enrollment (use of DXA testing for the purpose of osteoporosis screening). ${ }^{12}$ Exclusion criteria were (1) residing at a longterm acute care hospital, skilled nursing facility, adult foster home, or assisted living facility and (2) either enrolled in hospice care or with an estimated life expectancy of $<6$ months. The sampling strategy was systematic alphabetical sampling with a random start. ${ }^{14}$

Administrative claim data were collected from the Department of Medical Operations, which was unaware of the study objectives and were merged with clinical data by researchers. Researchers surveyed each participant's electronic medical record (EMR) from study enrollment every 90 days in chronologic order. Clinical data from EMRs were matched with administrative claim data by name and date of birth. To ensure participant confidentiality, all personal identifiers were removed from the study databases after matching was achieved. There was difficulty in interpreting the statistical analysis because at relatively small numbers ( $<5 \%$ of total participants) of Hispanics, Native Americans, and Asians, and we restricted our analyses to whites and blacks only and sensitivity analyses of alternative ethnic categories. ${ }^{15,16}$ Missing data accounted for $7 \%$ of data values and were most frequent for amount of education $(6 \%)$ and marital status (5\%). The number of participants meeting all eligibility criteria was 1320 .

\section{Main outcomes}

The main outcomes were (1) whether study participants received $B M D$ tests at least once during each study period and (2) where the BMD tests were performed. The method of BMD testing was DXA testing; the brand and model of DXA tests were not uniform. DXA testing sites were (1) an independent diagnostic testing facility or physician's office and (2) a hospital outpatient department. DXA testing included axial skeleton views of the hip, pelvis, and spine. Other forms of bone mass measurement (e.g., peripheral skeleton and quantitative computed tomography) were not considered, as they are less commonly performed and do not directly reflect the screening guidelines for osteoporosis., ${ }^{5,10,13}$ Osteoporosis prevalence during each period was measured; the definition of osteoporosis by DXA testing was a T-score of $\leq-2.5$. $^{2}$ Osteoporotic women were identified by chronic diseases, including generalized, postmenopausal, idiopathic, disuse, and other osteoporosis, using the International Classification of Diseases, 9th revision (ICD-9).

\section{Participant characteristics}

Participant characteristics were age, ethnicity, education, marital status, hazardous lifestyle, health insurance, comorbidities related to bone health, cognitive impairment, and home health or day care services. Hazardous lifestyle was defined as either current smoking or $>7$ units of alcohol/ week ( 1 unit $=14 \mathrm{~g}$ of alcohol=1 glass of wine $=1$ pint of beer $=1$ ounce of spirits). ${ }^{17,18}$ Supplemental private health insurance is additional out-of-pocket health insurance coverage beyond Medicare. ${ }^{11}$ Supplemental private health insurance included Medicare advantage plus but did not include dual eligibility for Medicare and Medicaid.

Comorbidities related to bone health included thyroid hormone supplement or history of hyperthyroidism, any anticonvulsant use, ever use of oral prednisone $(5 \mathrm{mg} /$ day for $>3$ months), any immune suppressants, aromatase inhibitor, antineoplastic chemotherapeutics, history of rheumatoid arthritis, and hyperparathyroidism. ${ }^{2,4}$ A previous study found that persons with cognitive impairment were less likely to use the DXA test. ${ }^{19}$ To adjust the effects of cognitive impairment on DXA testing, cognitive information was captured by researchers from either the diagnostic code or the problem list in EMRs for Alzheimer's disease, vascular dementia, dementia with Lewy bodies, dementia with parkinsonism, Pick's disease, senile degeneration of the brain, cerebral degeneration, uncomplicated senile dementia, dementia not otherwise specified, and cognitive disorder not otherwise specified. Home health or day care services reflected the need for caregivers for physically impaired participants. BMD testing was reported to be also inversely associated with physical disability. ${ }^{19}$

For ease of interpretation, characteristics were categorized as age (65-79 and $\geq 80$ ), ethnicity (white and black), education (high school education or less and high school graduation or higher), marital status (married and never married/widowed/divorced), supplemental private health insurance (yes and no), comorbidities related to bone health (yes and no), cognitive impairment (yes and no), and home health or day care services (yes and no). ${ }^{16}$ The study was approved by the institutional review board of Cleveland Clinic Health System. Because data were generated by reviewing medical records, informed consents were waived.

\section{Statistical analysis}

Bivariate comparisons of participant characteristics and measured osteoporosis prevalence between study phases were examined using chi-square tests to compare categorical data. All reported $p$ values were two-sided throughout, and $p<0.05$ was considered statistically significant. We used Cox proportional hazards regression models with the BMD test as the dependable variable to determine the predicted probability of the BMD test. After adjusting for independent variables (participant characteristics) by stepwise selection, we performed Cox proportional hazard regressions of the BMD test including data from all study participants and then performed separate regression analyses using data with or without supplemental private health insurance. Backward and forward selections were examined to ensure that the methods used for the selection of variables had no impact on analysis results. Adjusted hazard ratios (HR) of BMD tests 
along with corresponding $95 \%$ confidence intervals (CIs) were derived after adjusting for independent variables. HR $>1$ indicated that the predicted probability of the BMD test after reimbursement reductions was higher than that before reimbursement reductions. Sensitivity analyses were used to explore an alternative category of ethnicity (whites vs. blacks vs. others). Results for this alternative category are similar to those of the original category of ethnicity (whites vs. blacks) and are not included here. All data procedures and analyses were performed using SAS statistical software version 9.2 (SAS Institute, Cary, NC).

\section{Results}

\section{Participant characteristics and main outcomes}

Of the total participants $(n=1320)$, the numbers before (phase 1) and after (phase 2) reimbursement reductions were 647 and 673, respectively. Table 1 shows the participant characteristics and the main outcomes by study phase. Women aged $\geq 80$ comprised $42 \%$ and $45 \%$ of the group in phases 1 and 2 , respectively. Blacks accounted for $56 \%$ in phase 1 and $58 \%$ in phase 2. High school graduation or those with higher education represented $43 \%$ and $47 \%$ in phases 1 and 2, respectively. Those who never married, were widowed, or were divorced accounted for $35 \%$ and $38 \%$ in phases 1 and 2, respectively. Current smokers and excessive drinkers, representing women with a hazardous lifestyle, were $12 \%$ and $11 \%$ in phases 1 and 2 , respectively. In phase 1, 67\% had supplemental private health insurance, and $69 \%$ in phase 2 had supplemental private health insurance. In phase 1,14\% had the comorbidities related to bone health, and in phase $2,16 \%$ had comorbidities related to bone health. Women with cognitive impairment comprised $15 \%$ and $13 \%$ of the cohort in phases 1 and 2 , respectively. Those with home health or day care services constituted $24 \%$ and 20\% in phases 1 and 2, respectively. Osteoporosis prevalence was $14 \%$ in phase 1 and $15 \%$ in phase 2 .
For the main outcomes, $26 \%$ and $27 \%$ of participants received BMD tests at least once during phases 1 and 2, respectively. Among participants receiving BMD tests in phase $1(n=168)$, BMD tests performed at the site of (1) an independent diagnostic facility or physician's office, (2) a hospital outpatient department, and both (1) and (2) were 71\%, 16\%, and $13 \%$, respectively. Among participants receiving BMD tests in phase $2(n=182), B M D$ tests performed at the site of (1) an independent diagnostic facility or physician's office, (2) a hospital outpatient department, and both (1) and (2) comprised $62 \%, 23 \%$, and $15 \%$, respectively. All participant characteristics and the main outcomes did not differ significantly by study phases except for the study sites of BMD tests $(p=0.02)$

\section{Predicted probability of BMD test: Associations between study phases and BMD testing in overall participants and subsets by health insurance}

Table 2 presents the results of Cox proportional hazards regression analyses to determine the predicted probability of a BMD test with the associations between study phases and BMD testing in overall participants and subsets by health insurance. For those without supplemental private health insurance $(n=421)$, the adjusted probability of a BMD test in phase $2(19 \%, 95 \%$ CI $16 \%-22 \%)$ was lower than that in phase 2 $(24 \%, 95 \%$ CI $21 \%-27 \%$; HR $=0.67,95 \%$ CI $0.34-0.98, p=0.03)$. In the overall participants $(n=1320)$ and those with supplemental private health insurance $(n=899)$, there was no significant difference in the predicted probability of BMD testing by study phase. Stepwise and backward selections did not change the results of multivariate regressions. There was no evidence of multicollinearity or significant interactions between the variables in regressions, including data from all study participants and subsets by health insurance status. ${ }^{15}$ The regressions were assessed with goodness-of-fit testing procedures, which confirmed that these were not violated. ${ }^{15}$

Table 1. Participant Characteristics and Main Outcomes by Study Phases

\begin{tabular}{|c|c|c|c|}
\hline Characteristics, \% (n) & $\begin{array}{l}\text { Phase } 1, \mathrm{n}=647 \\
\text { Before reimbursement cuts }\end{array}$ & $\begin{array}{l}\text { Phase } 2, \mathrm{n}=673 \\
\text { After reimbursement cuts }\end{array}$ & $\mathrm{p}^{\mathrm{a}}$ \\
\hline Age, $\geq 80$ & $42(272)$ & $45(302)$ & 0.41 \\
\hline Ethnicity, black & $56(362)$ & $58(390)$ & 0.57 \\
\hline High school graduate or more & $43(279)$ & $47(316)$ & 0.28 \\
\hline Never married, widowed, or divorced & $35(227)$ & $38(256)$ & 0.36 \\
\hline Hazardous lifestyle & $12(77)$ & $11(75)$ & 0.13 \\
\hline Health insurance; supplementary private insurance & $67(434)$ & $69(465)$ & 0.68 \\
\hline Comorbidities related to bone health & $14(91)$ & $16(108)$ & 0.47 \\
\hline Cognitive impairment & $15(97)$ & $13(87)$ & 0.63 \\
\hline Home health or day care services & $24(156)$ & $20(134)$ & 0.12 \\
\hline Osteoporosis prevalence & $14(91)$ & $15(101)$ & 0.62 \\
\hline Main outcomes, \% (n) & $\begin{array}{l}\text { Phase } 1, \mathrm{n}=647 \text { Before } \\
\text { reimbursement cuts }\end{array}$ & $\begin{array}{l}\text { Phase } 2, \mathrm{n}=673 \text { After } \\
\text { reimbursement cuts }\end{array}$ & $\mathrm{p}^{\mathrm{a}}$ \\
\hline Number of participants receiving BMD test at least once & $26(168)$ & $27(182)$ & 0.59 \\
\hline Among participants receiving BMD test at least once & Phase $1, n=168$ & Phase $2, n=182$ & \\
\hline \multicolumn{4}{|l|}{ Study sites } \\
\hline A. Independent diagnostic facility or physician's office & $69(116)$ & $62(113)$ & 0.02 \\
\hline B. Hospital outpatient department & $18(30)$ & $23(42)$ & \\
\hline Both $\mathrm{A}$ and $\mathrm{B}$ & $13(22)$ & $15(27)$ & \\
\hline
\end{tabular}

$p$ values were derived from bivariate comparisons of participant characteristics and the main outcomes by study phase. $\mathrm{BMD}$, bone mineral density. 
Table 2. Predicted Probability of Bone Mineral Density Test: Associations Between Study Phase and Bone Mineral Density Testing in Overall Participants and Subsets by Health Insurance

\begin{tabular}{|c|c|c|c|c|}
\hline \multirow[b]{2}{*}{ Participant population } & \multicolumn{2}{|c|}{$\begin{array}{c}\text { Predicted probability of BMD } \\
\text { test, } \%(95 \% \text { CI })\end{array}$} & \multirow{2}{*}{$\begin{array}{l}\text { Hazard ratio } \\
\quad(95 \% \text { CI })\end{array}$} & \multirow[b]{2}{*}{$\mathrm{p}$} \\
\hline & Phase 1 & Phase 2 & & \\
\hline Overall participants $(n=1320)$ & $\begin{array}{l}26(21-32) \\
n=647\end{array}$ & $\begin{array}{l}27(22-33) \\
n=673\end{array}$ & $1.04(0.62-1.49)$ & 0.64 \\
\hline \multicolumn{5}{|l|}{ Subset by health insurance } \\
\hline $\begin{array}{l}\text { Participants with supplementary private insurance } \\
\quad(n=899)\end{array}$ & $\begin{array}{l}28(24-32) \\
\quad n=434\end{array}$ & $\begin{array}{l}30(26-35) \\
n=465\end{array}$ & $1.08(0.71-1.52)$ & 0.41 \\
\hline $\begin{array}{l}\text { Participants without supplementary private insurance } \\
\quad(n=421)\end{array}$ & $\begin{array}{l}24(21-27) \\
n=213\end{array}$ & $\begin{array}{l}19(16-22) \\
n=208\end{array}$ & $0.67(0.34-0.98)$ & 0.03 \\
\hline
\end{tabular}

${ }^{a}$ Hazard ratios were derived from Cox proportional hazard regressions after adjusting for participant characteristics (age, ethnicity, education, marital status, hazardous lifestyle, health insurance, comorbidities related to bone health, cognitive impairment, and home health or day care services). Hazard ratio $>1$ indicates the predicted probability that the BMD test in phase 2 was higher than that in phase 1.

CI, Confidence interval.

\section{Discussion}

Although the overall number of BMD tests was not affected after the reimbursement reductions, the results indicated that certain groups without supplemental private health insurance used the BMD test at a significantly lower rate since the reimbursement reductions. Consistent with nationally representative reports on osteoporosis screening for older adults, less than a third of study participants in the present study had a DXA test in 3 years. ${ }^{8,9,13}$ BMD testing rates in the present and prior studies are much lower than those of other screening services for older women, such as mammography, where two thirds of eligible populations were tested within the past 2 years. ${ }^{3,20}$ BMD testing rates for the purpose of osteoporosis prevention may vary by patient demographics. For example, although performed at academic health centers similar to those in the present study, BMD testing rates performed at facilities with predominantly white population are substantially higher $(64 \%-70 \%$ in the past 3 years) than those in the present study. ${ }^{19,21}$

Consistent with prior investigations of sites that perform DXA tests, the shift in BMD testing from nonhospital settings (e.g., physicians' offices and independent diagnostic facilities) to hospital settings was observed in the present study. 8,10 Several lines of evidence reflecting physicians' attitudes toward the reimbursement cuts in DXA testing have shown that more than a third of physicians reduced the number of DXA tests and opted to stop investing in DXA machines for their offices. $^{8,9,12,22}$ Reimbursement reductions for DXA tests in nonhospital settings appear to be closely linked to the physicians' attitudes. Although the Affordable Care Act of 2010 partially restored Medicare payment during 2010 and 2011, the shift in DXA test sites is expected to continue., 822

To our knowledge, the present study is the first to examine the effects of supplemental private health insurance on the relationship between reimbursement reductions for DXA testing and the BMD testing rate for female Medicare beneficiaries. Prior studies have shown that suboptimal DXA testing was associated with lower use of osteoporosis treatment, which suggests that those who carry supplemental health insurance may be better able to afford osteoporosis management options. ${ }^{5,11,23}$ Having supplemental health insurance in addition to Medicare may make it easier for Medicare beneficiaries to afford their various healthcare needs related to osteoporosis (e.g., more frequent physician office visits, switching from generic to brand name medications) because of lower copayments than those Medicare-only beneficiaries might be expected to pay.

The use of DXA testing as an osteoporosis screening tool has been reported to be a marker for interest level in osteoporosis management. ${ }^{1,2,11}$ The decreased use in DXA tests may raise concerns that patients and physicians are paying little attention to osteoporosis management. Although Medicare covers DXA testing every 2 years (or more frequently if necessary), DXA testing was more accessible to beneficiaries with supplemental health insurance than to those without such insurance.

Our findings highlight the urgent need for public attention to resolve the disparity in access to osteoporosis screening by health insurance type; similar disparities in diverse public health services across acute and chronic disease care have been reported. ${ }^{23-25}$ In addition, preventing osteoporotic fracture through DXA testing in older women has been shown to be associated with lower likelihood of nursing home admission, higher quality of life, and reductions in public financial burden. Therefore, the resulting decrease in BMD testing because of a lack of supplemental private health insurance is likely to cause a lower quality of care. ${ }^{2-4,8,12,22}$

We acknowledge several limitations to the study design. Because data collection was limited in a U.S. urban area and distinct racial composition (blacks representing more than half of the participants), the study cannot be generalized to other areas or populations. Furthermore, the use of secondary data may have led to observer variation in clinical data from EMRs. About $7 \%$ of data were missing, and data from other health systems and physician characteristics were not included in the analysis; therefore, selection bias may have occurred. Other than health insurance status, information representing socioeconomic status (e.g., annual income) and physician characteristics were not included; for this reason, examining the internal consistency of our findings was also limited. Therefore, our findings should be interpreted with caution and considered preliminary until they are confirmed in future studies with more representative data.

\section{Conclusions}

We found a significant association between reimbursement reductions and decrease in BMD testing in female Medicare beneficiaries without supplemental private health insurance. 


\section{Acknowledgments}

This project was supported by the American Geriatrics Society Seed Grant Research Program. The funding source had no role in design and conduct of the study; collection, management, analysis, and interpretation of the data; and preparation, review, or approval of the manuscript. We thank Pil Park, Ph.D. (University of Michigan Medical School), and Daehyun Kim, M.D., M.P.H. (Harvard Medical School), for their valuable feedback on study design, analysis interpretation, and manuscript preparation.

\section{Disclosure Statement}

All authors have no financial and personal relationships with other people or organizations that could inappropriately influence (bias) their work.

\section{References}

1. Firestein GS, Kelly WN. Kelly's textbook of rheumatology, 8th ed. Philadelphia: Saunders/Elsevier, 2009.

2. National Osteoporosis Foundation (NOF). 2008 Clinician's guide to prevention and treatment of osteoporosis. Available at www.nof.org/professionals/clinicians_guide.htm Accessed May 7, 2012.

3. Halter JB, Studenski S, Oudlander JG, et al. Hazzard's geriatric medicine and gerontology, 6th ed. New York: McGraw-Hill, 2010.

4. Kanis JA, on Behalf of World Health Organization Scientific Group. Assessment of osteoporosis at the primary health care level. Technical report. WHO Collaborating Centre for Metabolic Diseases, University of Sheffield, UK, 2007. Available at www.shef.ac.uk/FRAX. Accessed May 7, 2012.

5. Center for Medicare and Medicaid Services (CMS). Bone mass measurement. In: Medicare Learning Network $₫$ : The guide to Medicare preventive services. (ICN 006439). Available at www.cms.gov/MLNproducts/downloads/ mps_guide_web-061305.pdf Accessed May 2, 2012.

6. Bonnick SL. Bone densitometry in clinical practice: Application and interpretation, 3rd ed. Totowa, NJ: Humana Press, 2010.

7. Department of Health and Human Services. Medicare coverage of and payment for bone mass measurements. Washington, DC: Federal Registry, 1998 34320-34328.

8. King AB, Fiorentino DM. Medicare payment cuts for osteoporosis testing reduced use despite tests' benefit in reducing fractures. Health Aff (Millwood) 2011;30:2362-2370.

9. O'Malley CD, Johnston SS, Lenhart G, et al. Trends in dualenergy x-ray absorptiometry in the United States, 2000-2009. I Clin Densitom 2011;14:100-107.

10. Zhang J, Delzell E, Zhao H, et al. Central DXA utilization shifts from office-based to hospital-based settings among Medicare beneficiaries in the wake of reimbursement changes. J Bone Miner Res 2012;27:858-864.

11. Cadarette SM, Gignac MA, Jaglal SB, Beaton DE, Hawker GA. Access to osteoporosis treatment is critically linked to access to dual-energy x-ray absorptiometry testing. Med Care 2007;45:896-901.

12. Hayes BL, Curtis JR, Laster A, et al. Osteoporosis care in the United States after declines in reimbursements for DXA. J Clin Densitom 2010;13:352-360.

13. Curtis JR, Carbone L, Cheng H, et al. Longitudinal trends in use of bone mass measurement among older Americans, 1999-2005. J Bone Miner Res 2008;23:1061-1067.

14. Hulley SB, Cummings SR, Browner WS, Grady D, Hearst N, Newman TB. Design clinical research, 2nd ed. Philadelphia: Lippincott/Williams \& Wilkins, 2001.

15. Hosmer DW, Lemeshow S. Applied logistic regression, 2nd ed. Hoboken, NJ: John Wiley \& Sons, 2005.

16. Stokes ME, Davis CS, Koch GG. Categorical data analysis using the SAS system, 2nd ed. Cary, NC: SAS Institute Inc, 2009.

17. National Institute on Alcohol Abuse and Alcoholism. The physician's guide to helping patients with alcohol problems (Publication NIH 95-3769). Washington, DC: Government Printing Office, 1995.

18. Saunders JB, Aasland OG, Babor TF, De La Fuente TR, Grant M. Development of the Alcohol Use Disorders Identification Test (AUDIT): WHO collaborate project on early detection of persons with harmful alcohol consumption. Addiction 1993;88:791-804.

19. McNally DN, Kenny AM, Smith JA. Adherence of academic geriatric practitioners to osteoporosis screening guidelines. Osteoporosis Int 2007;18:177-183.

20. Breen N, Gentleman JF, Schiller JS. Update on mammography trends: Comparisons of rates in 2000, 2005, and 2008. Cancer 2011;117:2209-2218.

21. Powell H, O'Connor K, Greenberg D. Adherence to the U.S. Preventive Services Task Force 2002 osteoporosis screening guidelines in academic primary care settings. J Womens Health 2012;21:50-53.

22. Tanner SB. Dual-energy $x$-ray absorptiometry in clinical practice: New guidelines and concerns. Curr Opin Rheumatol 2011;23:385-388.

23. Wilper AP, Woolhandler S, Lasser KE, McCormick D, Bor $\mathrm{DH}$, Himmelstein DU. A national study of chronic disease prevalence and access to care in uninsured U.S. adults. Ann Intern Med 2008;149:170-176.

24. Lewiecki EM, Baim S, Siris ES. Osteoporosis care at risk in the United States. Osteoporos Int 2008;19:1505-1509.

25. Sabik LM, Dahman BA. Trends in care for uninsured adults and disparities in care by insurance status. Med Care Res Rev 2012;69:215-230.

Address correspondence to: Ji Won Yoo, M.D., M.Sc. Department of Internal Medicine University of Michigan School of Medicine 300 North Ingalls Building, Room 932 Ann Arbor, MI 48109-2007

E-mail: yoojiw@trinity-health.org 